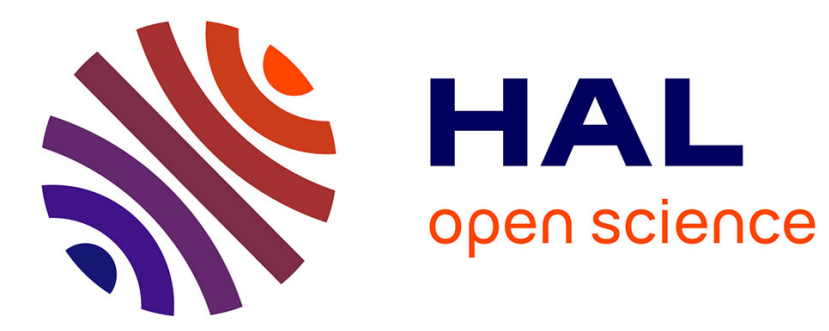

\title{
Un changement dans la continuité
}

Catherine Berthelon, Jean-louis Soubret

\section{To cite this version:}

Catherine Berthelon, Jean-louis Soubret. Un changement dans la continuité. RTS - Recherche Transports Sécurité, 2013, 2013 (01), pp.3-3. 10.4074/S0761898013001015 . hal-01670627

\section{HAL Id: hal-01670627 \\ https://hal.science/hal-01670627}

Submitted on 21 Dec 2017

HAL is a multi-disciplinary open access archive for the deposit and dissemination of scientific research documents, whether they are published or not. The documents may come from teaching and research institutions in France or abroad, or from public or private research centers.
L'archive ouverte pluridisciplinaire HAL, est destinée au dépôt et à la diffusion de documents scientifiques de niveau recherche, publiés ou non, émanant des établissements d'enseignement et de recherche français ou étrangers, des laboratoires publics ou privés. 


\section{Un changement dans la continuité}

\section{Change with continuity}

\section{Catherine Berthelon · Jean-Louis Soubret}

(c) IFSTTAR et Éditions NecPlus 2013

Chers lecteurs, chers auteurs, relecteurs et collègues

Suite à une réorientation de la politique de Springer vers des revues anglophones et à orientation médicale, nous avons été amenés à changer de maison d'édition pour la publication de Recherche Transports Sécurité.

Notre choix s'est porté sur les Éditions NecPlus, une maison indépendante dont l'objectif principal est de publier et de diffuser des revues scientifiques françaises et d'excellence en sciences humaines et sociales, qui bénéficient ou ont vocation à obtenir un facteur d'impact. Le site de NecPlus est développé, maintenu et hébergé par Cambridge University Press sur sa plateforme technologique : Cambridge Journals Online.

Les Éditions NecPlus publient au standard de diffusion en ligne international qui comprend notamment les caractéristiques suivantes :

- adhésion à CrossRef (résolveur de lien qui permet de savoir quelle référence bibliographique est citée dans quel article et de l'atteindre en cliquant sur un lien hypertexte) et attribution de DOI (Digital Object Identifier), l'identification numérique qui permet de retrouver un article ou toute autre unité documentaire en ligne ;

- statistiques d'utilisation des revues (norme COUNTER);

- identification par IP, Athens, Shibboleth et/ou mot de passe ;

- présence dans les grandes bases d'indexation internationales.
Grâce à ce nouvel environnement, Recherche Transports Sécurité devrait bénéficier d'un accroissement de son lectorat et d'une meilleure visibilité dans la communauté internationale francophone.

Ces changements n'affectent en rien la qualité scientifique et l'indépendance éditoriale du comité de rédaction de la revue. Rien ne change non plus en ce qui concerne le comité de rédacteurs et le comité éditorial international. Le suivi des articles n'est pas modifié puisque nous gardons le même site de soumission en ligne.

Dans ce contexte, nous envisageons toutefois à moyen terme de recentrer la revue sur le domaine des transports et de l'aménagement, et d'accepter des articles rédigés en anglais par des auteurs non francophones.

Ce premier numéro de l'année 2013 est composé d'articles originaux issus de recherches effectuées dans des domaines et disciplines variés. Il inclut également la seconde partie d'un dossier intitulé "Mobilité, transports et territoires dans la science régionale francophone : illustrations par quelques articles », dossier coordonné par Guy Joignaux et Lourdes Diaz Olvera qui en ont rédigé l'introduction.

Lecteurs, auteurs, relecteurs, nous vous remercions de votre niveau d'exigence et de votre fidélité renouvelée et nous vous incitons à continuer à faire connaître Recherche Transports Sécurité dans votre entourage scientifique.

Catherine Berthelon $(\bowtie)$

Directrice adjointe du Laboratoire Mécanismes d'accidents (LMA),

Département Transport Santé Sécurité (TS2)

Rédactrice en chef de Recherche Transports Sécurité

e-mail : catherine.berthelon@ifsttar.fr

Jean-Louis Soubret $(\square)$

Éditions NecPlus

56 rue de la Roquette, 75011 Paris - France

e-mail : jlsoubret@necplus.eu 